\title{
Automatic Pre-Identification Method of Navigation Tasks for Intelligent Ship
}

\author{
Jie Zhang", Qinglong Hao, and Ran Dai \\ Navigation College, Dalian Maritime University, Dalian, 116026, China
}

\begin{abstract}
In order to solve the problem of the large amount of real-time operation of the automatic identification method for intelligent ship navigation tasks, this paper analyzes the pattern characteristics of different navigation tasks, based on the information and data automatically perceived by ships, and uses large data processing analysis technology and the pick-up algorithm. The method of automatic pre-identification of intelligent ship navigation tasks is proposed, that is, the static pre-identification of each sub-navigation task of the planned route before sailing and the sharing of most real-time operations of automatic identification of navigation tasks. It lays the foundation for dynamic and accurate identification of navigation tasks. The simulation results show that the automatic pre-recognition method is feasible and the results have high reliability.
\end{abstract}

Keywords: navigation task; automatic pre-identification; pattern characteristics; pick-up algorithm

(Submitted on November 12, 2018; Revised on December 10, 2018; Accepted on January 6, 2019)

(C) 2019 Totem Publisher, Inc. All rights reserved.

\section{Introduction}

In 2016, the China Classification Society introduced the "Intelligent Ship Code", which sets relevant technical standards for intelligent ships and promotes domestic research on intelligent ship-related technologies. "Intelligent processing and display of navigation information for navigation tasks" is an important technology for ships to realize intelligence, and it can automatically provide appropriate information support for different navigation tasks. According to the practical experience of navigation, different navigation tasks, such as mooring, anchoring, and entering and leaving port, require different information support. To ensure that navigation information can be accurately matched for navigation tasks, the ship system should have the function of intelligent identification of navigation tasks [1].

The research on the new intelligent ship is in its initial stage now. There are few research results in the direction of intelligent identification of navigation tasks, and related issues such as the timeliness of intelligent identification remain to be solved. In the paper "Intelligent Identification Method for Navigation Tasks Based on Navigation Information", it is emphasized that based on shipborne navigation information and shore-based support information during navigation, a large number of real-time operations such as "initial judgment", "secondary judgment", and "accurate identification" are adopted to achieve the purpose of real-time identification of navigation tasks [2]. Although this method can achieve real-time identification of navigation tasks, it is affected by many real-time operations, resulting in slow response speed of recognition and poor timeliness of subsequent "intelligent processing and display of navigation information for navigation tasks". It cannot meet the requirements of ship intelligence.

At present, in the research fields of unmanned ships on intelligent collision avoidance, autonomous navigation, and operations, most researchers conducted research based on "according to predetermined tasks, unmanned ships sailing on the surface of the water with accurate satellite positioning and their own sensing" [3-4]. Yu Shuhai studied autonomous navigation and navigation control and could automatically control unmanned boats to complete scheduled tasks [5]. In applications such as the waste cleaning task of the Suzhou River and the collection of water samples from the Hejiang

\footnotetext{
* Corresponding author.

E-mail address: zjdlmu@163.com
} 
Water Pollution Incident in Guangxi, small unmanned ships cruise independently according to set routes and complete the setting of navigation tasks before sailing, reducing the calculation of real-time identification navigation tasks during navigation and gaining good practical results [6].

Therefore, in view of the fact that there is a poor time-sensitive problem in real-time identification of navigation tasks in the previous research methods, based on the concept of "pre-set tasks" for unmanned ships, an automatic preidentification method for navigation tasks for intelligent ships is proposed. It makes full use of the data information in the planned routes and electronic charts, pre-identifies the navigation tasks before sailing, and undertakes most of the calculations of the "initial judgment" and "secondary judgment" of the intelligent identification navigation tasks.

\section{Basic Conditions of Automatic Pre-Identification of Navigation Tasks}

Usually, after receiving the voyage order, a detailed voyage plan that focuses on designing a reasonable planned route is drawn up onboard. The ship will sail along the planned route performing voyage tasks. When a ship is engaged in cargo transportation, it needs to go through a variety of navigation tasks from the port of departure to the port of destination, such as mooring, berthing and unberthing, entering and leaving port, inshore navigation, coastal navigation, and ocean navigation. In order to ensure the intelligent navigation, the ship should be able to operate intelligently or unmanned after receiving the voyage order. With regard to the automatic route design, many experts and scholars have conducted relevant research and exploration, and the paper does not elaborate on it. The paper focuses on intelligent pre-identification of navigation task pattern based on navigation information and data after the route is automatically generated [7].

\subsection{Route Planning}

In general, the ship should sail along the planned route, that is, perform the navigation tasks on the planned route. Therefore, the pre-identification of the navigation task is based on the planned route. The entire route is identified by the ship's navigation status and business logic segment by segment. In order to facilitate the pre-identification of navigation tasks, the system automatically designs the route and generates a route list based on the voyage order. The route list contains at least the waypoint number, the latitude and longitude of the waypoint, the accumulated distance, the remaining distance, and the heading.

\subsection{Data and Information of Electronic Chart}

It is very important to perceive, analyze, and judge the electronic chart data and information around the ship during the preidentification process.

The ENC adopted in ECDIS is a vector data structure, which is an absolute one-to-one correspondence between space and characteristics. Based on the characteristics of the object (such as shape, color, etc.) and its position (such as geographical coordinates), it is possible to determine the only object with the corresponding characteristics at a specific location. The data model of the IHO S-57 is a vector data structure that describes the object entity through the combination of space objects and feature objects. The space object describes the spatial position attribute of the entity. The IHO S-57 adopts a vector mode, that is, the attributes of the object are represented by points, edges, and surfaces. Feature objects are attributes that describe the type, nature, and characteristics of entities. Feature objects are based on the existence of spatial objects and use spatial data to express their location. Both of them form the basic elements of IHO S-57 object data by encoding [8].

Each ENC has a unique unit name consisting of an 8-bit character encoding and a 3-digit extension, of which the third is for navigation purposes, as shown in Table 1.

Table 1. The ENC data navigation purpose code

\begin{tabular}{|c|c|c|}
\hline Code & Navigation purpose & Scale \\
\hline 1 & Overview & $1: 1499999$ \\
\hline 2 & General & $1: 90000 \sim 1: 349999$ \\
\hline 3 & Coastal & $1: 22000 \sim 1: 89999$ \\
\hline 4 & Approach & $1: 4000 \sim 1: 21999$ \\
\hline 5 & Harbour & $>1: 4000$ \\
\hline 6 & Berthing & \\
\hline
\end{tabular}




\section{Pre-Identification of Navigation Tasks based on Pattern Feature Analysis}

The voyage plan should include the entire process from berth to berth, including the location of the pilot's boarding, that is, the route design must start from the berth to the end of the berth. On the basis of the planned route, combined with the relevant information and data of the route list and electronic charts, this paper mainly analyzes the characteristics of navigation tasks at each stage of navigation and summarizes its pattern recognition method.

A complete voyage involves many navigation tasks [9]. In general, its business logic is shown in Figure 1.

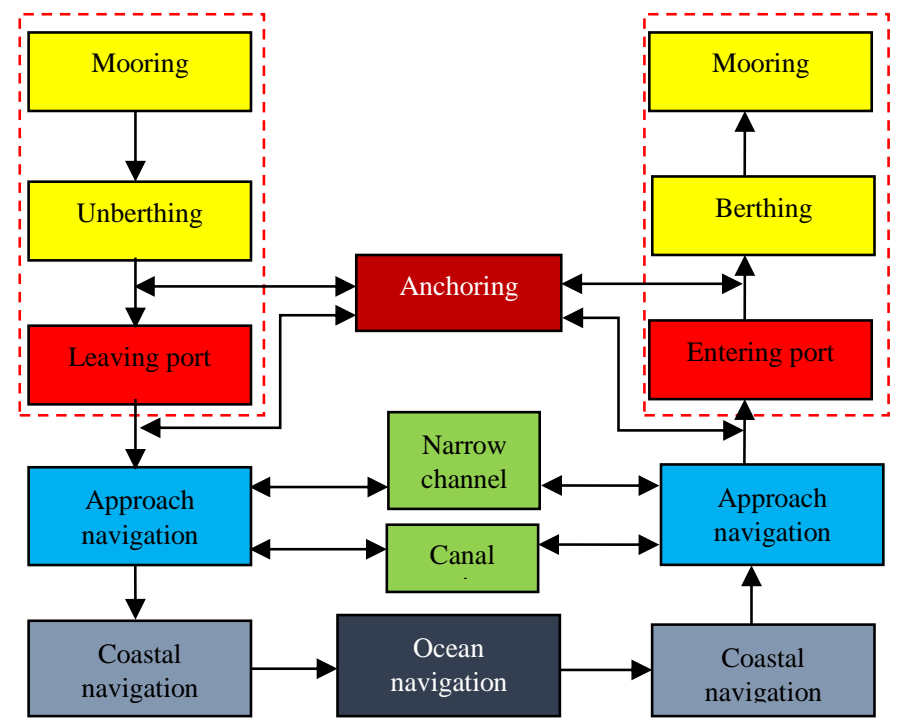

Figure 1. The logical business flow chart of a certain voyage

- Taking "mooring" as the starting point, the ship completes "unberthing" inside the harbor and "leaving port" to the pilot station, and "anchoring" may be involved in this process.

- Leaving the pilot station, the ship enters the stage of "inshore navigation", which may involve "narrow channel navigation" or "canal navigation". Then, it gradually enters the waters of "coastal navigation" and "ocean navigation".

- Before approaching the destination port, the ship goes through "ocean navigation", "coastal navigation", and "inshore navigation" until arrival the pilot station, followed by "entering port" and "berthing". "Mooring" is taken as the terminal point.

- If there are more than two ports of call, the "mooring, unberthing and berthing, leaving and entering port" business will appear repeatedly.

Pre-identification methods for typical navigational tasks are as follows.

\subsection{Pre-Identification Method for "Mooring"}

\subsubsection{Feature Analysis}

The ship is at port of call during the "mooring", "berth" information can be picked up near the waypoint, the third of the unit name of the largest scale chart near the waypoint is 6 , the accumulated distance is 0 at the departure port (WP1), and the remaining distance is 0 at the destination (WPn).

\subsubsection{Pre-Identification Method}

According to the feature of accumulated distance at departure port (0) and remaining distance at destination (0), "mooring" can be identified at these two positions. Their waypoints are marked as " $\mathrm{P}_{\text {mooring-start }}$ " and " $\mathrm{P}_{\text {mooring-end" }}$ " respectively.

For any route, the presence of a middle port of call and its location are not fixed, so the distance cannot be used as the basis for the judgment of "mooring" at the middle port of call. Its judgment method is shown in Figure 2. 


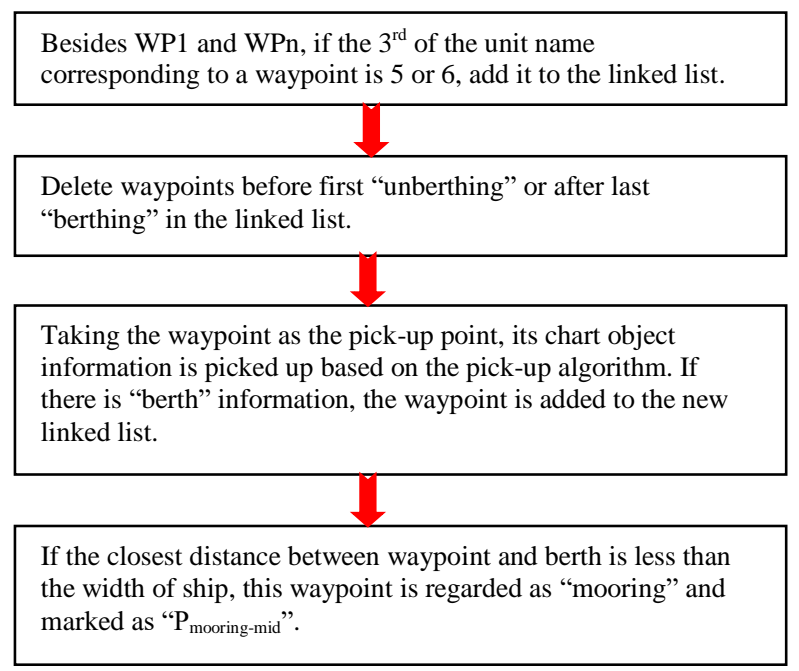

Figure 2. The judgement flowchart of "mooring" at middle port of call

\subsubsection{Relevant Algorithm}

The algorithm for distance between the pick-up point and the point, line, or plane is shown in Figure 3.

Definition: the coordinates of pick-up point $M$ is $\left(x_{M}, y_{M}\right)$, the reference point for the point element $T$ is $\left(x_{T}, y_{T}\right)$, the axis of the linear element $L$ is fold line $\mathrm{O}_{0} \mathrm{O}_{1} \mathrm{O}_{2} \cdots \mathrm{O}_{n}$, and the regional element is defined by its closed boundary fold line [10].

The distance from pick-up point $M$ to point element $T$ is defined as the distance between point $M$ and $T$. It is defined as Equation (1):

$$
d(M, T)=\sqrt{\left(x_{M}-x_{T}\right)^{2}+\left(y_{M}-y_{T}\right)^{2}}
$$

The distance from pick-up point $M$ to linear element $T$ is defined as the distance from point $M$ to its axis. It is defined as Equation (2):

$$
d(M, L)=\frac{\min }{i=0, n-1} d\left(M, \overline{O_{\imath} O_{l+1}}\right)
$$

In Equation (2), $\mathrm{d}\left(M, \overline{O_{\mathrm{i}} O_{\mathrm{i}+1}}\right)$ is the distance from point $M$ to line segment $\overline{O_{\mathrm{i}} O_{\mathrm{i}+1}}$. It is defined as Equation (3):

$$
d\left(M, \overline{O_{l} O_{l+1}}\right)=\frac{\min }{P \in O_{i} O_{i+1}} d(M, P)
$$

The distance between pick-up point $M$ and point $P$ is $\mathrm{d}(M, P)$.

The distance from the pickup point $M$ to the regional element is defined as the distance from $M$ to its boundary line. In order to calculate $\mathrm{d}\left(M, \overline{O_{\mathrm{i}} O_{\mathrm{i}+1}}\right)$, a vertical line is drawn from the point $M$ to the straight line $O_{\mathrm{i}} O_{\mathrm{i}+1}$, and the vertical foot is $P$. If $P$ is in the line segment $\overline{O_{\mathrm{i}} O_{\mathrm{i}+1}}$, the distance of the straight segment $M P$ is the distance from $M$ to the straight line $O_{\mathrm{i}} O_{\mathrm{i}+1}$. It is defined as Equation (4):

$$
d\left(M, \overline{O_{l} O_{l+1}}\right)=\frac{\left|\left(x_{M}-x_{i}\right)\left(y_{i+1}-y_{i}\right)-\left(y_{M}-y_{i}\right)\left(x_{i+1}-x_{i}\right)\right|}{\sqrt{\left(x_{i+1}-x_{i}\right)^{2}+\left(y_{i+1}-y_{i}\right)^{2}}}
$$

If $P$ is outside the line segment $\overline{O_{\mathrm{i}} O_{\mathrm{i}+1}}$, the smaller distance from $M$ to the ends of the line segment is the distance from $M$ to the straight line $O_{\mathrm{i}} O_{\mathrm{i}+1}$. It is defined as Equation (5):

$$
d\left(M, \overline{O_{l} O_{l+1}}\right)=\min \left\{d\left(M, O_{i}\right), d\left(M, O_{i+1}\right)\right\}
$$




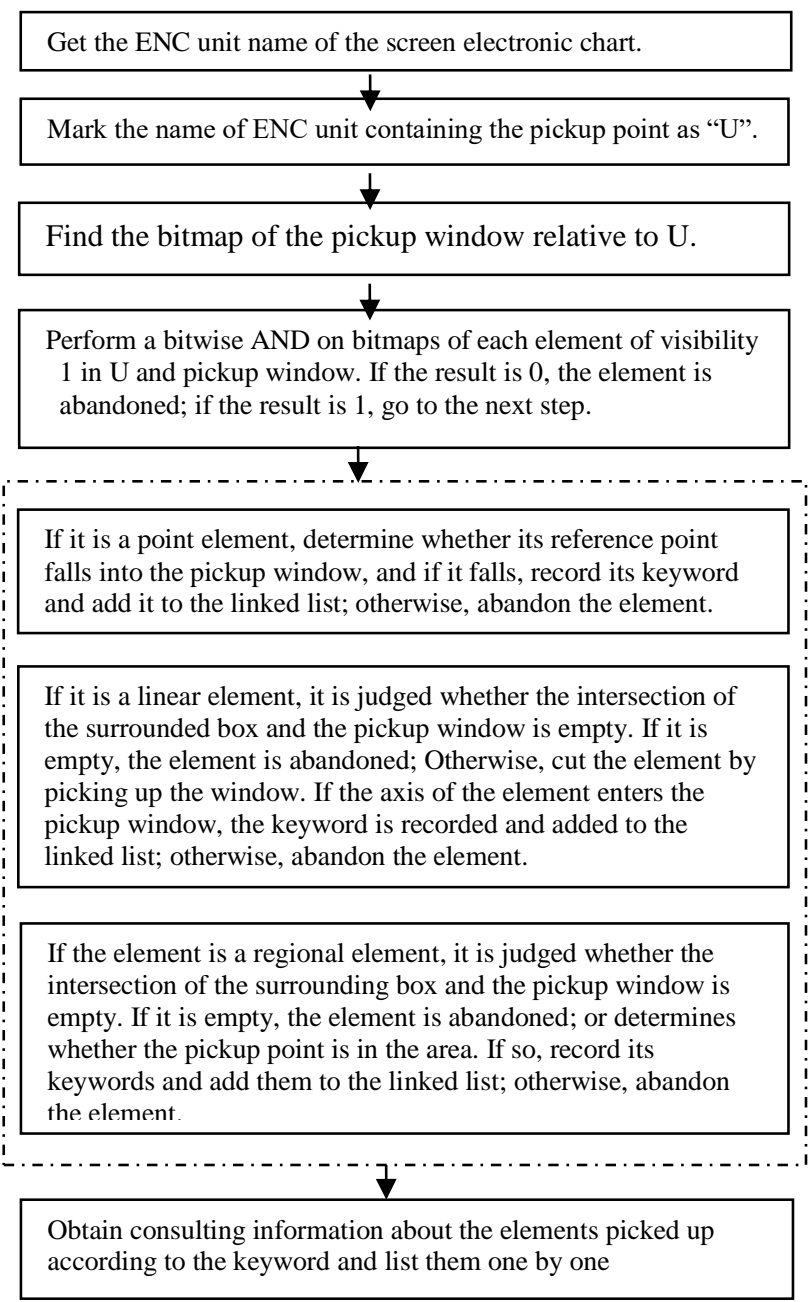

Figure 3. The flowchart of pickup algorithm for ENC element

To determine the relationship between point $P$ and line segment $\overline{O_{\mathrm{i}} O_{\mathrm{i}+1}}$, list the equation of straight line $O_{\mathrm{i}} O_{\mathrm{i}+1}$ and vertical line $M P$.

$$
\begin{gathered}
O_{i} O_{i+1}:\left\{\begin{array}{l}
y-y_{i}=t_{1}\left(y_{i+1}-y_{i}\right) \\
x-x_{i}=t_{1}\left(x_{i+1}-x_{i}\right)
\end{array}\right. \\
M P:\left\{\begin{array}{c}
y-y_{M}=t_{2}\left(x_{i+1}-x_{i}\right) \\
x-x_{M}=-t_{2}\left(y_{i+1}-y_{i}\right)
\end{array}\right.
\end{gathered}
$$

Based on the simultaneous Equations (6) and (7), the result is shown in Equation (8) at point $P$.

$$
t_{1}=\frac{\left(x_{i+1}-x_{i}\right)\left(x_{M}-x_{i}\right)+\left(y_{i+1}-y_{i}\right)\left(y_{M}-y_{i}\right)}{\left(x_{i+1}-x_{i}\right)^{2}+\left(y_{i+1}-y_{i}\right)^{2}}
$$

If $0 \leq t_{1} \leq 1$, point $P$ is in the line segment $\overline{O_{\mathrm{i}} O_{\mathrm{i}+1}}$; otherwise, it is outside the line segment $\overline{\mathrm{O}_{\mathrm{i}} O_{\mathrm{i}+1}}$.

\subsection{Pre-Identification Method for "Berthing" and "Unberthing"}

\subsubsection{Feature Analysis}

In general, after "berthing" or before "unberthing", the ship is in the situation of "mooring".

"Design code of general layout for sea ports" stipulates that usually the ship's berthing waters take 2B (B is the width 
of the ship), the roundabout waters take 2 to $3 \mathrm{~L}$ ( $\mathrm{L}$ is the length of the ship), and the braking waters take 3 to $5 \mathrm{~L}$ [11].

Based on the simulation test results of nearly 5,000 ships operating under different types and tons of ships under various winds and currents, using SPSS statistical analysis, the water area required for ship berthing or unberthing with a cumulative frequency of $95 \%$ or more is within the range of $5 \mathrm{~L}$ from berth. Therefore, combining the code and a large number of measured data, the ship is berthing or unberthing within the range of $5 \mathrm{~L}$ from "mooring" state.

\subsubsection{Pre-Identification Method}

The first waypoints more than 5L before and after "mooring" are noted as $\mathrm{P}_{\text {berthing }}$ and $\mathrm{P}_{\text {unberthing }}$ respectively. The ship is "unberthing" in the sections of $\mathrm{P}_{\text {mooring-start }}-\mathrm{P}_{\text {unberthing }}$ and $\mathrm{P}_{\text {mooring-mid }}-\mathrm{P}_{\text {unberthing}}$; it is "berthing" in the sections of $\mathrm{P}_{\text {mooring-end }}$ $\mathrm{P}_{\text {berthing }}$ and $\mathrm{P}_{\text {mooring-mid }}-\mathrm{P}_{\text {berthing }}$.

\subsection{Pre-Identification Method for "Entering or Leaving Port"}

\subsubsection{Feature Analysis}

In view of the complexity of the navigation environment in the port waters, in order to ensure the safety of the port and ships, ships entering and leaving the port are usually operated under the command of the pilot. During entering or leaving port, the pilot leads the ship between the pilot station and the berth. Therefore, from the point of view of the planned route, "entering or leaving port" is the in the section between the pilot station and the berth (except for mooring and berthing operations in the port) [12].

\subsubsection{Pre-Identification Method}

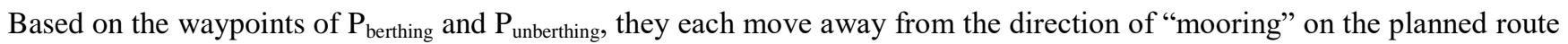
and pick up their surrounding object information one by one at the waypoints until they pick up the "pilot station" information where the waypoint is the boundary point for navigation entering or leaving port. Among them, the waypoint after $\mathrm{P}_{\text {unberthing }}$ is denoted as $\mathrm{P}_{\text {Pilot-out }}$, and the waypoint before $\mathrm{P}_{\text {berthing }}$ is denoted as $\mathrm{P}_{\text {Pilot-in. }}$. $\mathrm{P}_{\text {Pilot-out }}$ and $\mathrm{P}_{\text {Pilot-in }}$ appear in pairs. The navigation task of the $\mathrm{P}_{\text {unberthing }}-\mathrm{P}_{\text {Pilot-out }}$ section is "leaving port", and the navigation task of the $\mathrm{P}_{\text {Pilot-in }}-\mathrm{P}_{\text {berthing }}$ section is "entering port" [13].

\subsection{Pre-Identification Method for "Inshore Navigation"}

After leaving the pilot station or before entering the pilot station, the ship navigates inshore. During inshore navigation, the navigation tasks that ships may normally perform are divided into narrow channel navigation, canal navigation, and general inshore navigation. When navigating inshore, the third of the unit name of the largest scale chart near the waypoint is greater than 3 [14].

\subsubsection{Pre-Identification Method for "Narrow Channel Navigation"}

Narrow channels usually refer to navigable waters that are narrow in width and limited in the operation of ships. In fact, it is difficult to quantify exactly what the width of navigable waters is to be considered as a narrow channel, and there has been no uniform international standard for many years. It is customary internationally to consider waterways less than two nautical miles in width as narrow channels. Therefore, when a ship is sailing in the navigable waters with a limited width, if the width of the navigable waters on either side of the passage plan is less than one nautical mile, the water area is considered to be a narrow channel, that is, the ship performs "narrow waterway navigation" [10].

All waypoints between any set of waypoints of $\mathrm{P}_{\text {Pilot-out }}$ and $\mathrm{P}_{\text {Pilot-in }}$ must perform the following judgment procedure if the third of the unit name of the largest scale chart near the waypoint is greater than 3 .

For the segment that needs to be judged, a route domain is established that is a simple rectangular of one nautical mile each to the left and right of the route, as shown in Figure 4. Taking 1.2 times of the ship's draft as safety depth, extraction of sounding in the route domain smaller than the safety depth, and corresponding spatial and geographical information, such as latitude and longitude, find the four boundary points in which latitude and longitude are extreme values (the position of the asterisk in Figure 4). Then, make a vertical line to the segment to obtain four vertical feet $(A, B, C, D$ in Figure 4$)$, and the segment $(A-D)$ between the outermost two points is "narrow channel navigation". The rest $(A-W P X, D$ - WPY $)$ are "inshore navigation" [15]. 


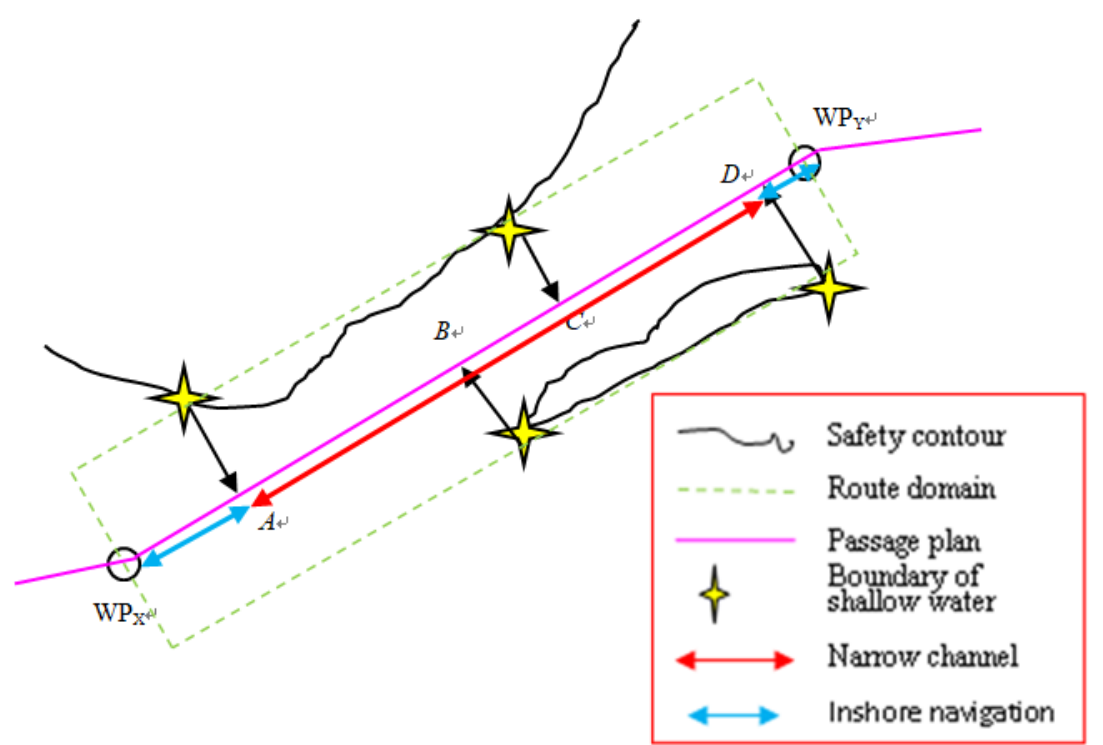

Figure 4. The pre-identification for navigation in narrow channels

\subsubsection{Pre-Identification for "Canal Navigation"}

In general, the ship is compulsory to the pilot in canals. Before entering a canal, the ship should enter the pilot station at sea near the entrance and then navigate in the canal, finally leaving another pilot station at sea near the outlet.

Based on the element information picked from the above waypoints, all waypoints that are usually a continuous set of waypoints containing canal information are extracted, and the waypoints at both ends of this set of waypoints are used as the basic points to view the information of their respective outer route points. Find the waypoints containing information of the

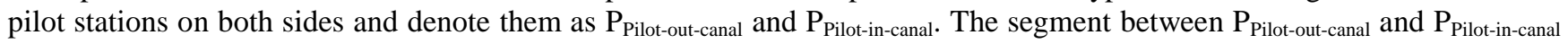
is "canal navigation".

\subsubsection{Pre-Identification for Other "Inshore Navigation"}

In the inshore navigation area, if a ship does not perform the navigation tasks of "narrow channel navigation" and "canal navigation", it defaults to "inshore navigation".

\subsection{Pre-Identification Method for Other Navigation Tasks}

Coastal navigation and ocean navigation depend on the scale of the chart at the location of the waypoint. If the third of the unit name of the largest scale chart near the waypoint is 3 , it is "coastal navigation". If the third of the unit name of the largest scale chart near the waypoint is 2 , it is "ocean navigation".

\section{Verification of Pre-Identification Method of Navigation Task}

\subsection{Experiments and Results}

Using MATLAB 2010b to design the pre-identification program of navigation task, the feasibility and correctness of the research method are verified. The pre-identification of a route from Dalian to Qingdao is shown in Figure 5.

The results of pre-identification are shown in Table 2.

\subsection{Discussion}

Through the manual comparison, this route has a total of 18 waypoints, and only the WP12 identification result is inconsistent. The pre-identification method is generally feasible, and the identification results are basically correct. The reason for the inconsistent identification result of WP12 is that the identification reference for "entering or leaving port" in the identification method is the location of the pilot station. The fairway of Qingdao port is longer, and the location of the 
pilot station is not at the entrance of the fairway, as shown in Figure 5(c). Instead, it is located at a point about ten nautical miles from the entrance of the fairway, causing identification errors. Therefore, the next step should take into account the characteristics of "fairway" to improve the identification method for "entering or leaving port".

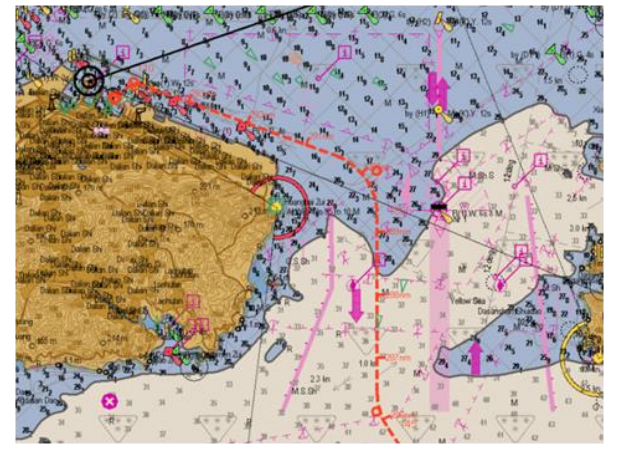

(a) Waters near Dalian port

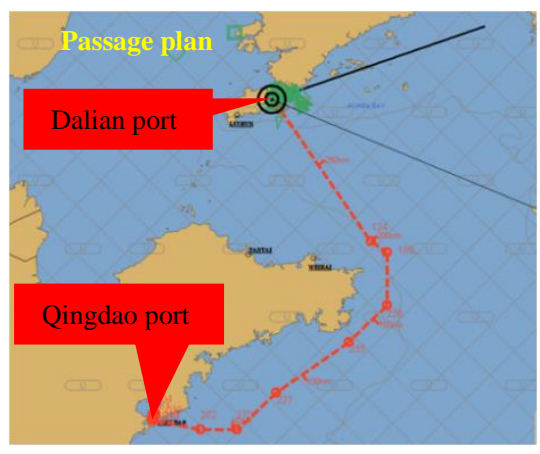

(b) Passage Plan

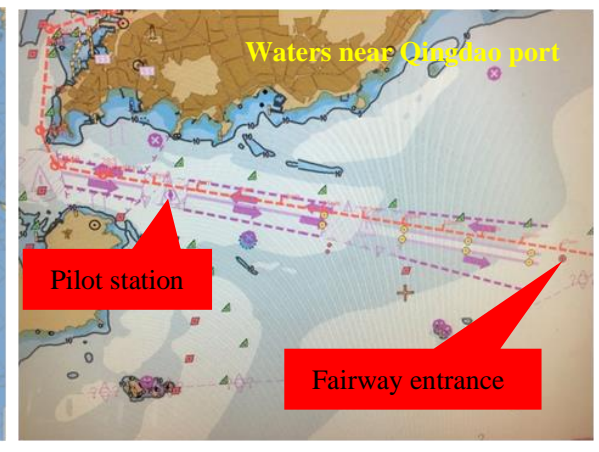

(c) Waters near Qingdao port

Figure 5. The pre-identified route

Table 2. The pre-identification results

\begin{tabular}{|c|c|c|c|}
\hline \multirow{2}{*}{ Waypoint } & \multicolumn{3}{|c|}{ Navigation task } \\
\hline & Identification & Real & Comparison \\
\hline WP1 & Mooring & Mooring & $\sqrt{ }$ \\
\hline WP2 & Unberthing & Unberthing & $\sqrt{ }$ \\
\hline WP3 & Leaving & Leaving port & $\sqrt{ }$ \\
\hline WP4 & Inshore navigation & Inshore navigation & $\sqrt{ }$ \\
\hline WP5 & Coastal navigation & Coastal navigation & $\sqrt{ }$ \\
\hline WP6 & Coastal navigation & Coastal navigation & $\sqrt{ }$ \\
\hline WP7 & Coastal navigation & Coastal navigation & $\sqrt{ }$ \\
\hline WP8 & Coastal navigation & Coastal navigation & $\sqrt{ }$ \\
\hline WP9 & Coastal navigation & Coastal navigation & $\sqrt{ }$ \\
\hline WP10 & Inshore navigation & Inshore navigation & $\sqrt{ }$ \\
\hline WP11 & Inshore navigation & Inshore navigation & $\sqrt{ }$ \\
\hline WP12 & Inshore navigation & Entering port & $x$ \\
\hline WP13 & Entering port & Entering port & $\sqrt{ }$ \\
\hline WP14 & Entering port & Entering port & $\sqrt{ }$ \\
\hline WP15 & Entering port & Entering port & $\sqrt{ }$ \\
\hline WP16 & Entering port & Entering port & $\sqrt{ }$ \\
\hline WP17 & Berthing & Berthing & $\sqrt{ }$ \\
\hline WP18 & Mooring & Mooring & $\sqrt{ }$ \\
\hline
\end{tabular}

\section{Conclusions}

Taking passage plan and electronic charts as sources of information and analyzing the features of various navigation tasks, pre-identification methods for maritime tasks are formulated. After verification, the results of recognition are correct. Based on the idea of distributing real-time computing and making full use of effective information resources, this method focuses on pre-identification of navigation tasks before sailing and does not study the entire process of identification, so identification errors are inevitable. Due to the limitations of information sources, navigation tasks cannot be divided finely by this method. In order to achieve accurate navigation task identification, information sources should be further expanded. Through the pre-identification of navigation tasks before sailing, most of the calculations of intelligent identification navigation tasks are shared, which will play a good role in the intelligent identification of real-time navigation tasks. On this basis, we should further explore the integration of multi-source information, such as dynamic navigation environment information and shore-based information perceived by intelligent ships, to provide technical support for "intelligent processing and display of navigation information for navigation tasks".

\section{Acknowledgements}

This work was partly supported through grants from the National Natural Science Foundation of China (No. 51679025), Natural Science Foundation of Liaoning Province (No. 20170540093), and Research Funds for the Central University (No. 3132018151,3132018152 ). The authors would like to thank the anonymous reviewers for their valuable comments. 


\section{References}

1. R. Kuang, "A Kind of Ship Target Detection and State Recognition Algorithm," Science Mosaic, Vol. 176, No. 7, pp. 4-7, 2016

2. J. Zhang, J. J. Feng, and X. W. Yue, "Intelligent Identification Method for Navigation Tasks based on Navigation Information," Ship and Ocean Engineering, Vol. 46, No. 2, pp. 185-187, 2017

3. J. Hu, "Research on Unmanned Ship Autopilot System based on Human Simulated Intelligent Control," Ship Science and Technology, Vol. 39, No. 14, pp. 46-48, 2017

4. H. Q. Shen, C. Guo, T. S. Li, and Y. L. Tu, "An Intelligent Collision Avoidance and Navigation Approach of Unmanned Surface Vessel Considering Navigation Experience and Rules," Journal of Harbin Engineering University, Vol. 39, No. 6, pp. 998-1005, 2018

5. S. H. Yu, "Integrated Design and Application of Autonomous Navigation and Navigation Control for Unmanned Craft," Unmanned Carrier and Control Technology Development and Application Academic Discussion Summit Collection, Vol. 39, No. 14, pp. 46-48, 2017

6. J. C. Jin, W. L. Cui, J. Zhang, and L. E. Sun, "Water Sampling from Reservoirs in Offshore Districts using an Unmanned Surface Vehicle," Marine Sciences, Vol. 42, No. 1, pp. 11-14, 2018

7. Z. J. Gao, Y. J. Zhang, P. T. Sun, and W. H. Li, "Research Summary of Unmanned Ship," Journal of Dalian Maritime University, Vol. 43, No. 2, pp. 1-7, 2017

8. J. P. Zhang, "Electronic Chart Display and Information System," Dalian Maritime University, pp. 6-38, 2014

9. L. Yue, G. Xiang, and W. H. Hu, "A Mission-Oriented Simulating and Optimizing Method for the Shipborne System Information Flow," Chinese Journal of Ship Research, Vol. 10, No. 6, pp. 114-119, 2015

10. Y. J. Zhang, "Mathematics and Algorithm Fundamentals of Electronic Chart," Dalian Maritime University, pp. 76-135, 2001

11. X. L. Bian, et al., "Design Code of General Layout for Sea Ports," Ministry of Transport, pp. 18-50, 2013

12. K. Wiltold and S. Andrzej, "Radar and Automatic Identification System Track Fusion in an Electronic Chart Display and Information System," Journal of Navigation, Vol. 68, No. 6, pp. 1141-1154, 2015

13. D. A. Zilberbr, J. A. Kaminka, and H. Zarosim, "Fast and Complete Symbolic Plan Recognition," in Proceedings of the 19th International Joint Conference on Artificial Intelligence, pp. 653-658, 2005

14. K. Shehzad, U. Akram, and S. Razzaq, "Behavior Recognition Using Multivariate M-Mediod based Modeling of Motion Trajectories," Multimedia Systems, Vol. 21, No. 5, pp. 485-505, 2015

15. G. Christopher and R. P. Goldman, "A Probabilistic Plan Recognition Algorithm based on Plan Tree Grammars," Artificial Intelligence, Vol. 173, No. 11, pp. 1101-1132, 2009

Jie Zhang graduated from the Navigation College at Dalian Maritime University with a bachelor's degree and master's degree. His current research interests include intelligent ship, E-navigation, and intelligent information processing.

Qinglong Hao graduated from the Navigation College at Dalian Maritime University with a bachelor's degree and master's degree. His current research interests include intelligent ship and intelligent information processing.

Ran Dai graduated from the Navigation College at Dalian Maritime University with a bachelor's degree and master's degree. His current research interests include intelligent ship, navigation safety evaluation, and intelligent information processing. 